\title{
Study on the collapsibility sensitivity of loess in different acid environment by consolidometer
}

\author{
Yao Zhang ${ }^{1,2}$, Cheng $\mathrm{Jin}^{2}$, Ning $\mathrm{Li}^{2, \mathrm{a}}$, Zaiqiang $\mathrm{Hu}^{2}$ \\ 1Shaanxi Key Laboratory of Safety and Durability of Concrete Structures, Xijing University, Xi'an 710123, China. \\ ${ }^{2}$ School of Civil Engineering and Architecture, Xi'an University of Technology, Xi'an 710048, China.
}

\begin{abstract}
Cement connection of loess is an important internal factor of loess collapse, which has a direct impact on the sensitivity of the collapse. In this study, different concentrations of nitric acid were added into the immersion water solution of loess collapsibility test. The collapsibility deformation and stability time were measured under different pressure and acid environment. The test results show that: in acid solution, the collapsibility coefficient of collapsible loess increases more under low pressure, and the collapsibility stability time is prolonged under all levels of pressure; Loess has different collapsibility sensitive pressure range in aqueous solution and acidic solution. The structural strength of collapsible loess has an important influence on the depth of collapsible loesr. It is very important to the constructions on the loess area
\end{abstract}

\section{Introduction}

As a quaternary sediment, loess is widely distributed in the world, accounting for about one tenth of the land area. China is the country with the widest and deepest loess distribution in the world, mainly distributed in the Loess Plateau in Northwest China, North China Plain and the south of Northeast China, covering an area of 631000 square kilometers, accounting for about $6 \%$ of the land area of the whole country [1] [2]. Loess has a unique property - collapsibility, which is different from other sediments in the same period, that is, loess collapses rapidly after being soaked by water under self weight or external load. This characteristic of collapsible loess often brings serious damage to the geotechnical engineering built on this kind of loess, such as large-area cracking and subsidence of foundation and subgrade, inclination and collapse of high-rise structures such as houses, etc. . The degree of damage caused by loess collapsibility is not only related to the collapsibility of loess, but also related to the collapsibility sensitivity of loess. The sensitivity of loess collapsibility is generally considered as the difficulty, speed and size of loess collapsibility after water immersion. It is different from collapsibility of loess, but they are related to each other. The Loess with high collapsibility may have weak collapsibility sensitivity, and the Loess with low collapsibility may have stronger collapsibility sensitivity than the Loess with high collapsibility, so the damage may be greater than that of the Loess with high collapsibility. Therefore, the study of loess collapsibility sensitivity is as important as the study of loess collapsibility, which is of great significance to the engineering construction in loess area. However, there are few studies on loess collapsibility sensitivity at home and abroad and there is no method to evaluate loess collapsibility sensitivity that can be widely recognized and promoted.

The fundamental reason of loess collapsibility lies in its unique internal material composition and structural characteristics. Microscopic observation shows that loess is composed of structural units (single ore body, aggregate and agglomerate), cements (clay, organic matter and calcium carbonate) and pores (macropores, overhead pores and intergranular pores). When the loess is infiltrated by water, the strength of salt crystal cementation will be weakened, and it will lose stability under a certain pressure, resulting in other micro structural units around the pores falling into the pores . Therefore, salt crystal cementation (including insoluble salt, moderately soluble salt and easily soluble salt) plays an important role in loess collapsibility, which largely determines the difficulty, speed and size of loess collapsibility, that is, the collapsibility sensitivity of loess.

The fundamental reason of loess collapsibility lies in its unique internal material composition and structural characteristics. Microscopic observation shows that loess is composed of structural units (single ore body, aggregate and agglomerate), cements (clay, organic matter and calcium carbonate) and pores (macropores, overhead pores and intergranular pores). When the loess is infiltrated by water, the strength of salt crystal cementation will be weakened, and it will lose stability under a certain pressure, resulting in other micro structural units around the pores falling into the pores [3]. Therefore, salt crystal cementation (including insoluble salt, moderately soluble salt and easily soluble salt) plays an important role in loess collapsibility, which largely determines the difficulty, speed and size of loess collapsibility, that is, the collapsibility sensitivity of loess.

\footnotetext{
a Corresponding author: ningli@xaut.edu.cn
} 
Because salt crystal cementation belongs to microstructure, it is difficult to test its mechanical properties by conventional mechanical tests. Therefore, there are few experimental studies on the effect of salt crystal cementation on loess collapsibility and collapsibility sensitivity at home and abroad. In order to understand the mechanism of salt crystal cementation in loess collapsibility and collapsibility sensitivity, this paper uses the characteristic that nitric acid can rapidly dissolve salt crystal cementation, adds different concentrations of nitric acid into the immersion solution of loess collapsibility test, and tests the collapsibility sensitivity indexes of loess, such as collapsibility coefficient, stability time and collapsibility rate, under different acidic environments and different pressures [4] [5].

\section{Test details}

\subsection{Test programme}

In order to achieve the above research purposes, in the acid collapsibility test and conventional collapsibility samples, the collapsibility stability time and collapsibility coefficient are mainly investigated and analyzed. In order to further analyze the test phenomenon and prove the conclusion, the scanning observation of the microstructure of typical soil samples before and after the test was carried out by using the electronic microscope equipment

(1) The basic physical indexes of soil samples and the content of calcium carbonate are determined according to the relevant provisions of SL237-1999, so as to ensure that the calcium carbonate of soil samples can be fully dissolved in $2 \mathrm{~mol} / \mathrm{L}$ nitric acid solution.

(2) Preparation of solutions with different concentrations:

Calculate the amount and mass of nitric acid needed for a certain volume of $0.3 \mathrm{~mol} / 1$ and $2 \mathrm{~mol} / \mathrm{L}$ nitric acid solution, weigh it in a conical flask or other containers and configure it.

(3) Collapsibility test of different concentrations of solution

1) Twenty one $50 \mathrm{~cm} 2$ ring knife samples were divided into three groups. Each group of 7 samples were pressurized to $25,50,100,200,400,800$ and $1600 \mathrm{kpa}$ respectively until the deformation was stable (the settlement per hour was not more than $0.01 \mathrm{~mm}$ ).

2) After the above samples are pressurized and stabilized, distilled water, $0.3 \mathrm{~mol} / 1$ and $2 \mathrm{~mol} / \mathrm{L}$ nitric acid solutions are injected into the water injection tank of the consolidometer respectively, and the values are recorded every half an hour according to $0.10(6 \mathrm{~S}), 0.25$ (15s), 0.5 (30s), 1.00, 2.25, 4.00, 6.25, 9.00, 12.25, 16.00, $20.25,25.00,30.25,36.00,42.25,49.00$ and $64.00 \mathrm{~min}$, Until the deformation is stable (the subsidence per hour is not more than $0.01 \mathrm{~mm}$ ).

\subsection{Samples Properties}

The sampling site of this test is a construction foundation pit in the northern suburb of Xi'an city. The soil depth is $3.8-6.5 \mathrm{~m}$, which is Malan loess. The average collapsibility coefficient is 0.057 , which is the Loess with medium collapsibility.

\section{Test results and analysis}

\subsection{Comparison of collapsibility rate}

The collapsibility rate of loess refers to the change of collapsibility in different time periods, which can be expressed by the following formula, where hn and $\mathrm{h}(\mathrm{n}-1)$ are the collapsibility at corresponding time points, in $\mathrm{mm}$; $\mathrm{TN}$ and $\mathrm{t}(\mathrm{n}-1)$ are the corresponding time points, in min.

$$
v=\frac{h_{n}-h_{(n-1)}}{t_{n}-t_{(n-1)}}
$$

Fig. 1 shows the collapsibility rates of different concentrations of solutions under different pressures for 20 minutes. The comparison shows that the collapsibility rate of soil samples soaked in $2 \mathrm{~mol} / \mathrm{L}$ nitric acid is fast and lasts for a long time at $100 \mathrm{kPa}$ and $200 \mathrm{kPa}$, which is more sensitive (at $50 \mathrm{kPa}$, the collapsibility rate is not very fast before 20 minutes, but the collapsibility rate after 20 minutes is faster than that under other conditions), and the collapsibility rate of soil samples soaked in $0.3 \mathrm{~mol} / \mathrm{L}$ nitric acid is more sensitive at $200 \mathrm{kPa}$ and $400 \mathrm{kPa}$, Distilled water is more sensitive at $200 \mathrm{kPa}, 400 \mathrm{kPa}$ and $800 \mathrm{kPa}[4][5]$.

The analysis shows that: comparing the soil samples immersed in the same solution, it is found that each solution has a more sensitive pressure section. Below this pressure section, the hydraulic coupling effect is not enough to destroy the cementitious material in the soil sample during collapsibility, and the soil sample can not be fully compressed. Above this pressure section, the cemented connection of soil sample has been destroyed before immersion, which makes the later collapsible space small. This view is consistent with the conclusion of some other researchers. Jiang, M. J. believes that before the structural yield pressure of saturated soil, the collapsible deformation is very small, after the structural yield pressure of saturated soil, the collapsible deformation speed up, after the structural yield pressure peak of unsaturated soil, the collapsible deformation begins to decrease [6]. The content and composition of salt crystal cementation affect the location of this area. 


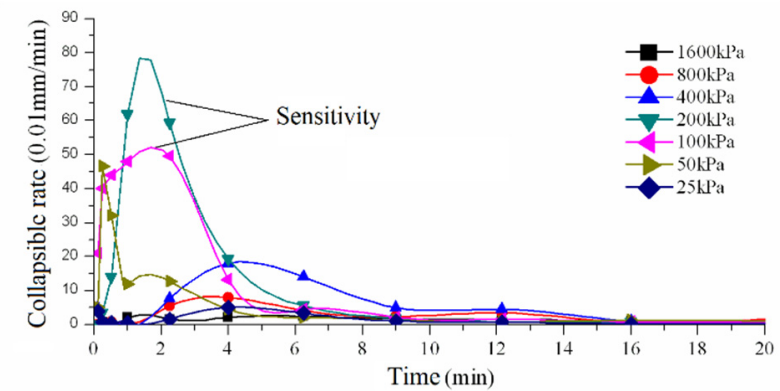

(a)Distilled water

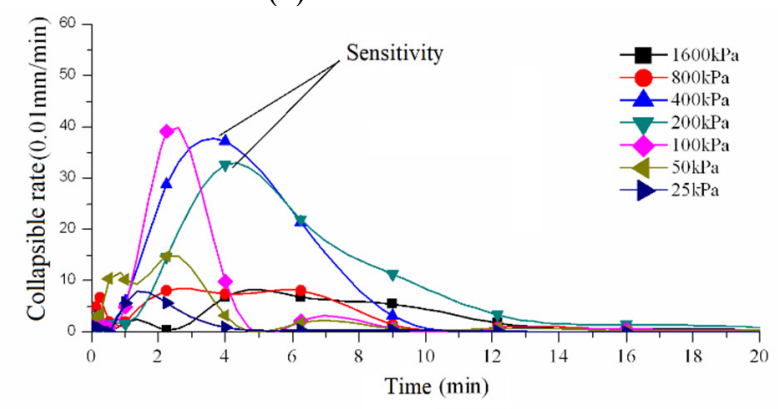

(b) $0.3 \mathrm{~mol} / \mathrm{L} \mathrm{HNO}_{3}$

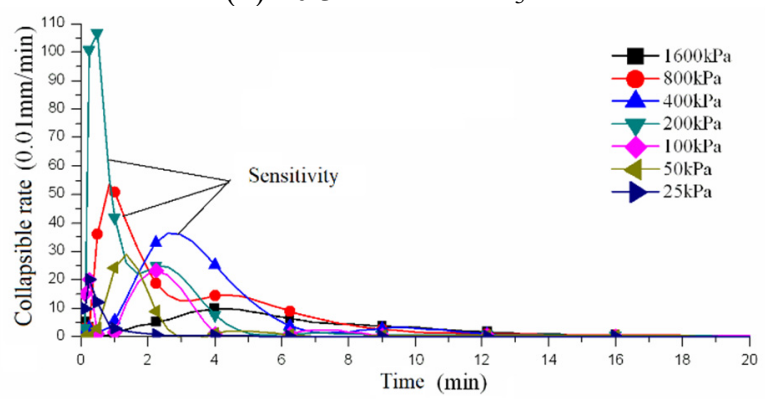

(c) $2 \mathrm{~mol} / \mathrm{L} \mathrm{HNO} 3$

Fig 1. Relationship between collapsible deformation rate and time in different solutions at different vertical pressures

\section{2 comparison of collapsible deformation}

Fig. 2 is a graph of collapsible deformation varying with time. It can be seen from Fig. 2 (a) that the collapsible deformation of the sample immersed in distilled water increases from $25 \mathrm{kpa}$ to $400 \mathrm{kPa}$, and decreases at $400 \mathrm{kPa}$ and $1600 \mathrm{kpa}$. In the range of $25 \mathrm{kpa}$ to $400 \mathrm{kPa}$, the collapsible deformation increases with the increase of pressure, because under low pressure, the strength of cemented connection before immersion can support the upper load. However, after immersion, the cementation formed by salt gradually dissolves in the water. The matric suction also decreases rapidly, which leads to the decrease of skeleton strength and the difficulty of bearing the upper load, resulting in the rapid failure of the structure.

The results show that the soil particles slide to the hole, the space between particles decreases, and the soil sample is compressed. With the increase of pressure, the connection between soil particles is easier to be destroyed and the soil sample is easier to be compressed. At 400$1600 \mathrm{kpa}$, the main reason for the reduction of collapsible deformation is that the structure of soil sample has been seriously damaged by higher additional pressure before immersion. Even the harder joints in soil (including cemented joints formed by insoluble salts such as calcium carbonate) are still difficult to resist this degree of load, and finally the soil sample has been fully compressed, However, the collapsible space becomes smaller in the later stage. This inference can be fully proved from the physical parameters of the soil sample after compression stability at all levels of pressure in Table 2 . When the pressure in Table 2 increases from 25 to $200 \mathrm{KPa}$, the dry density of the soil sample changes from 1.32 to 1.34 , the void ratio changes from 1.06 to 1.02 , and the compression coefficient changes from 0.011 to 0.028 , but the change is not obvious at $400 \mathrm{kPa}$, reaching $1.40,0.94$ and 0.0698 and $1600 \mathrm{kpa}$ respectively.

The variation is obvious and all parameters reach the peak value. It can be concluded that the structure of the soil sample can be destroyed and the soil sample can be fully compacted when the strength of the cemented connection of the soil sample is higher than that caused by the pressure increase. Therefore, the strength of the cemented connection must be an important factor affecting the occurrence of collapsibility. Because of the low strength of the cemented connection, the upper part only needs a small load to collapse, otherwise it is difficult to collapse.

It can be seen from Fig. 2 (b) and (c) that when immersed in $2 \mathrm{~mol} / \mathrm{L}$ and $0.3 \mathrm{~mol} / 1$ nitric acid, the collapsible deformation increases from $25 \mathrm{kpa}$ to $200 \mathrm{KPa}$ in turn, $200 \mathrm{KPa}$ is the peak of collapsible deformation, and decreases from $200 \mathrm{KPa}$ to $1600 \mathrm{kpa}$ in turn. The reason for the earlier peak value of collapse deformation is shown in 3.2 comparison of collapse coefficient.

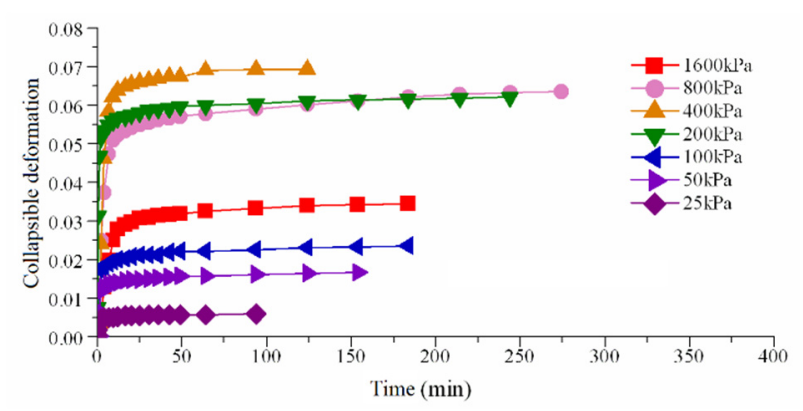

(a) Distilled water

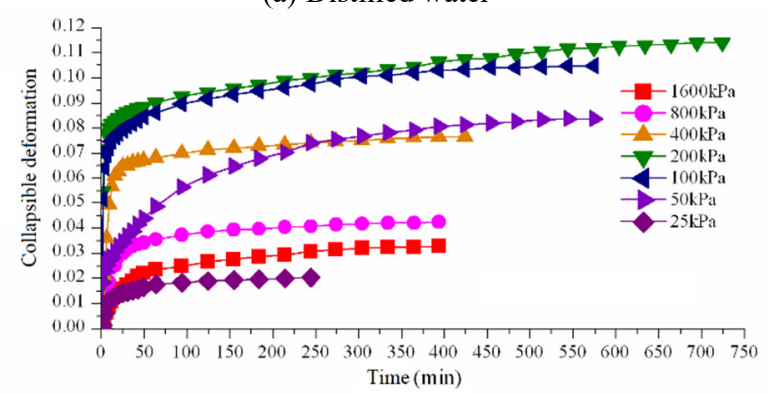

(b) $0.3 \mathrm{~mol} / \mathrm{L} \mathrm{HNO}_{3}$ 


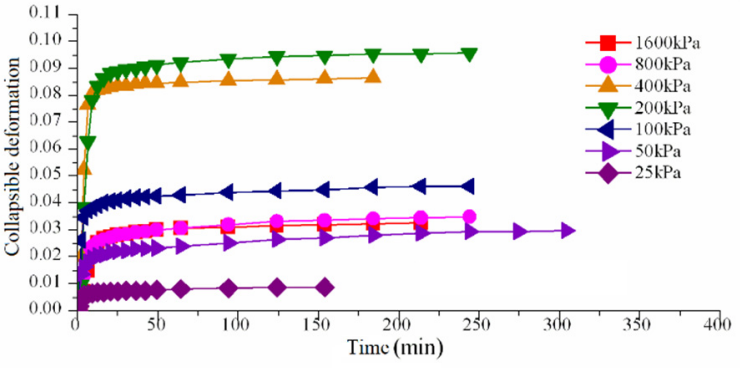

(c) $2 \mathrm{~mol} / \mathrm{L} \mathrm{HNO}_{3}$

Fig 2. Relationship between collapsible deformation and time at different vertical pressures

\subsection{Comparison of collapsible stability time}

In most cases, the stability time of $0.3 \mathrm{~mol} / 1$ nitric acid is slightly longer than that of distilled water. The main reason for this phenomenon is: after immersion in nitric acid, the nitric acid needs to have a certain reaction time with calcium carbonate and other insoluble salts in soil samples, and the reaction time is longer with the increase of nitric acid concentration. In this changing process, with the gradual dissolution of calcium carbonate and other salts by nitric acid, the colloidal connection strength gradually weakens. Under the same level of load, the soil sample will be slowly compressed until the reaction between nitric acid and calcium carbonate tends to be stable, and the upper load can not make the soil sample get significant deformation [9].

In situ site, because there is no acid to accelerate the dissolution of calcium carbonate, it often takes a long time to soak the insoluble salt such as calcium carbonate to be dissolved by the overlying pressure and water. Therefore, the insoluble salt such as calcium carbonate is also an important factor affecting the length of collapsibility time.

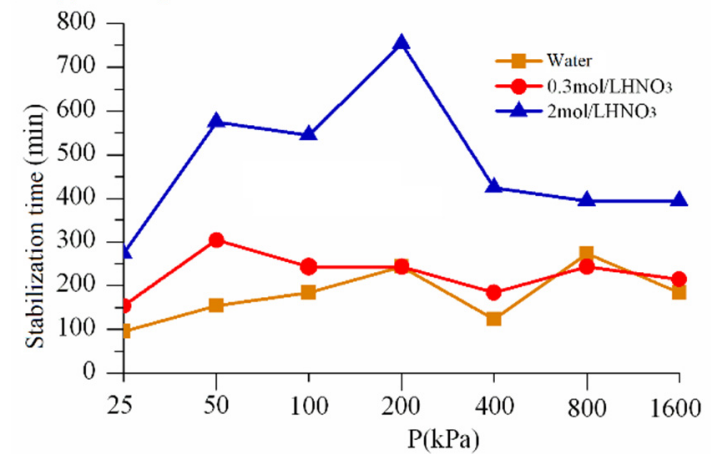

Fig 3. Comparison of stability time in different solutions at different vertical pressures

\section{Conclusion}

(1)Through the study of stability time, it is found that compared with distilled water solution, collapsible loess needs longer time to complete collapsible deformation in acid solution, because hydrogen ions in acid solution and insoluble salts such as calcium carbonate in Loess need sufficient time to react, thus prolonging the collapsible time.
(2)Through the study of deformation rate, it is found that the sensitive range of collapsible loess in acid solution and distilled water solution is different. Under the action of hydrogen ion, salt crystal cementation can be more easily decomposed, resulting in the collapsible sensitive pressure range of acid solution significantly reduced.

\section{Funding}

This work was supported by the China Postdoctoral Science Foundation (Grant No. 2020M673617XB), the Open Research Fund of State Key Laboratory of Geomechanics and Geotechnical Engineering, Institute of Rock and Soil Mechanics, Chinese Academy of Sciences (Grant No. Z020019), the special Foundation for High Level Talents of Xijing university (Grant No. XJ20B12).

\section{References}

1. Rao, S. M., Revanasiddappa, K. Collapse behaviour of a residual soil [J]. Geotechnique. 2002, 52 (52): 259-268.

2. $\mathrm{Hu}$ Zai-qiang, Shen Zhu-jiang, Xie Ding-yi. Deformation properties of structural loess [J]. Chinese Journal of Rock Mechanics and Engineering, 2004, 23(24): 4142-4146

3. $\mathrm{Hu}$ Zai-qiang, Shen Zhu-jiang, Xie Ding-yi. The structure of unsaturated loess [J]. Chinese Journal of Rock Mechanics and Engineering, 2000, 19(06): 775-779

4. Vilar1, O. M., Rodrigues, R. A. Collapse behavior of soil in a Brazilian region affected by a rising water table [J]. Canadian Geotechnical Journal, 2011, 48 (2): 226-233.

5. Casini, F. Deformation induced by wetting: a simple model [J]. Canadian Geotechnical Journal, 2012, 49 (49): 954-960.

6. Jiang, M. J., Hu, H. J., Liu, F. Summary of collapsible behaviour of artificially structured loess in oedometer and triaxial wetting tests $[\mathrm{J}]$. Canadian Geotechnical Journal, 2012, 49 (10): 1147-1157.

7. Gao, G. R. The distribution and geotechnical roperties of loess soils, lateritic soils and clayey soils in China [J] Engineering Geology, 1996, 42 (1): 95104.

8. Nouaouria, M. S., Guenfoud, M., Lafifi, B. Engineering properties of loess in Aleeria [J]. Engineering Geology. 2008, 99(1): 85-90.

9. Zhu Yuan-qing, Chen Zheng-han. A new method of studying collapsibility of loess [J]. Chinese Journal of Geotechnical Engineering 2008, 30 (4): 52 